\title{
Ductile Fracture Criteria in Prediction of Chevron Cracks
} Jindřich Petruška ${ }^{1, a}$, Petr Kubík ${ }^{1, b}$, Jiří Hůlka ${ }^{1, c}$ and František Šebek ${ }^{1, d}$

${ }^{1}$ Brno University of Technology, Brno, Technická 2896/2, 61669 Brno, Czech Republic

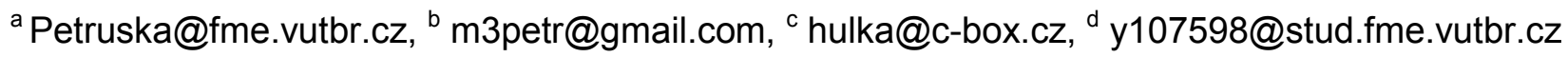

Keywords: Ductile fracture, Forward extrusion, FE simulation, Criteria calibration.

\begin{abstract}
Selected ductile fracture criteria are introduced and applied to prediction of chevron crack initiation and development during forward extrusion of long shafts. We present the calibration of selected criteria for carbon steel, simulate the process of forward extrusion and compare the simulation results with real experiments realized in a cooperating industrial company.
\end{abstract}

\section{Introduction}

Chevron fracture is a typical material damage occurring during forward extrusion of long products, especially after more consecutive reductions. It is dangerous due to its invisibility on the product surface. Process parameters such as die cone angle, area reduction ratio and friction have significant effect on the probability of chevron crack occurrence. Although successful analyses of the process using ductile fracture criteria and FE simulation have already been published [1,2], there are still many problems to be solved before the chevron bursting prediction and prevention will be a standard task of engineering design. This paper contains an analysis of the ability of selected fracture criteria to describe the influence of process parameters on the damage cumulation and crack initiation. All the analyzed criteria were implemented into ABAQUS/Explicit by user subroutine, their parameters calibrated from independent tensile tests and applied to extrusion simulation. In case of reaching critical value of damage, crack initiation and development are simulated by deleting appropriate finite elements.

\section{Ductile fracture criteria}

Four fracture criteria were used for chevron crack prediction, with stress triaxiality being the decisive variable

$$
\eta=\frac{\sigma_{m}}{\sigma_{e q}}
$$

where $\sigma_{\mathrm{m}}$ is the mean stress and $\sigma_{\mathrm{eq}}$ von Mises equivalent stress. The following criteria were applied:

Rice-Tracey criterion [3], defined by

$$
D=\frac{1}{1.65} \int_{0}^{\bar{\varepsilon}^{f}} \exp \left(\frac{3}{2} \eta\right) d \bar{\varepsilon}^{p}
$$

where the fracture initiates if the damage parameter $\mathrm{D}$ is equal to critical value $\mathrm{D}_{\mathrm{RT}}$.

RTCL criterion which is a combination of previous Rice-Tracey criterion with that of Cockroft-Latham, as presented by Törnqvist [4]:

$$
D=\int_{0}^{\bar{\varepsilon}^{f}} f(\eta)_{R T C L} d \bar{\varepsilon}^{p},
$$

where the weighting function $\mathrm{f}(\eta)$ is defined on three intervals

$$
f(\eta)_{R T C L}=\left\{\begin{array}{cl}
0 & \eta \in\left(-\infty,-\frac{1}{3}\right\rangle \\
2 \frac{1+\eta \sqrt{12-27 \eta^{2}}}{3 \eta+\sqrt{12-27 \eta^{2}}} & \eta \in\left(-\frac{1}{3}, \frac{1}{3}\right) \\
\frac{1}{1,65} \exp \left(\frac{3}{2} \eta\right) & \eta \in\left\langle\frac{1}{3}, \infty\right)
\end{array}\right.
$$

Again, the fracture occurs when the damage parameter $\mathrm{D}$ reaches critical value $\mathrm{D}_{\mathrm{RTCL}}$. 
Johnson-Cook criterion [5] in a simplified version without the influence of temperature and strain rate

$$
D=\int_{0}^{\bar{\varepsilon}^{f}} \frac{1}{\bar{\varepsilon}^{f}} d \bar{\varepsilon}^{p} \quad, \text { where } \bar{\varepsilon}^{f}=D_{1}+D_{2} \exp \left(D_{3} \eta\right)
$$

with three independent parameters $\mathrm{D}_{1}, \mathrm{D}_{2}, \mathrm{D}_{3}$.

Hyperbolic criterion, defined by

$$
D=\int_{0}^{\bar{\varepsilon}^{f}} \frac{\eta+E_{1}}{E_{2}} d \bar{\varepsilon}^{p}
$$

with two parameters $E_{1}, E_{2}$. According to the last two criteria the fracture evolution starts when the damage parameter $\mathrm{D}$ reaches unity.

\section{Criteria calibration}

There are two basic mechanisms of ductile failure of metals. Shear mechanism is prevailing for triaxiality close to zero, whereas growth and coalescence of voids is typical for tensile loading with large positive values of triaxiality. Chevron cracks initiation is caused by the second mechanism, generated at the symmetry axis in the process zone of extruded material. To describe the damage evolution under different stress-strain history represented by different stress triaxiality, four tensile test specimen types were used for fracture criteria calibration. Besides a standard smooth tensile specimen, three others with notch radii $5 \mathrm{~mm}, 2.5 \mathrm{~mm}$ and $1.2 \mathrm{~mm}$ were used (Fig.1). Multilinear model of stress-strain curve was used in all the simulations. To identify the flow curve, a trial-and-error strategy with the MLR correction as described in [6] was used. Experimental results of tensile tests and their computational simulations are compared in Figure 1 for all of the specimens. All the tests were realized for the Czech steel no. 412050 - equivalent of C60E4 (ISO) or C45 (DIN), with chemical composition given in Table 1.

Table 1 Chemical composition of the Czech steel no. 412050

\begin{tabular}{|l|l|l|l|l|l|l|l|l|}
\hline element & $\mathrm{C}$ & $\mathrm{Mn}$ & $\mathrm{Si}$ & $\mathrm{Cr}$ & $\mathrm{Ni}$ & $\mathrm{Cu}$ & $\mathrm{P}$ & $\mathrm{S}$ \\
\hline $\min [\%]$ & 0.42 & 0.50 & 0.17 & & & & & \\
\hline $\max [\%]$ & 0.50 & 0.80 & 0.37 & 0.25 & 0.30 & 0.30 & 0.04 & 0.04 \\
\hline
\end{tabular}
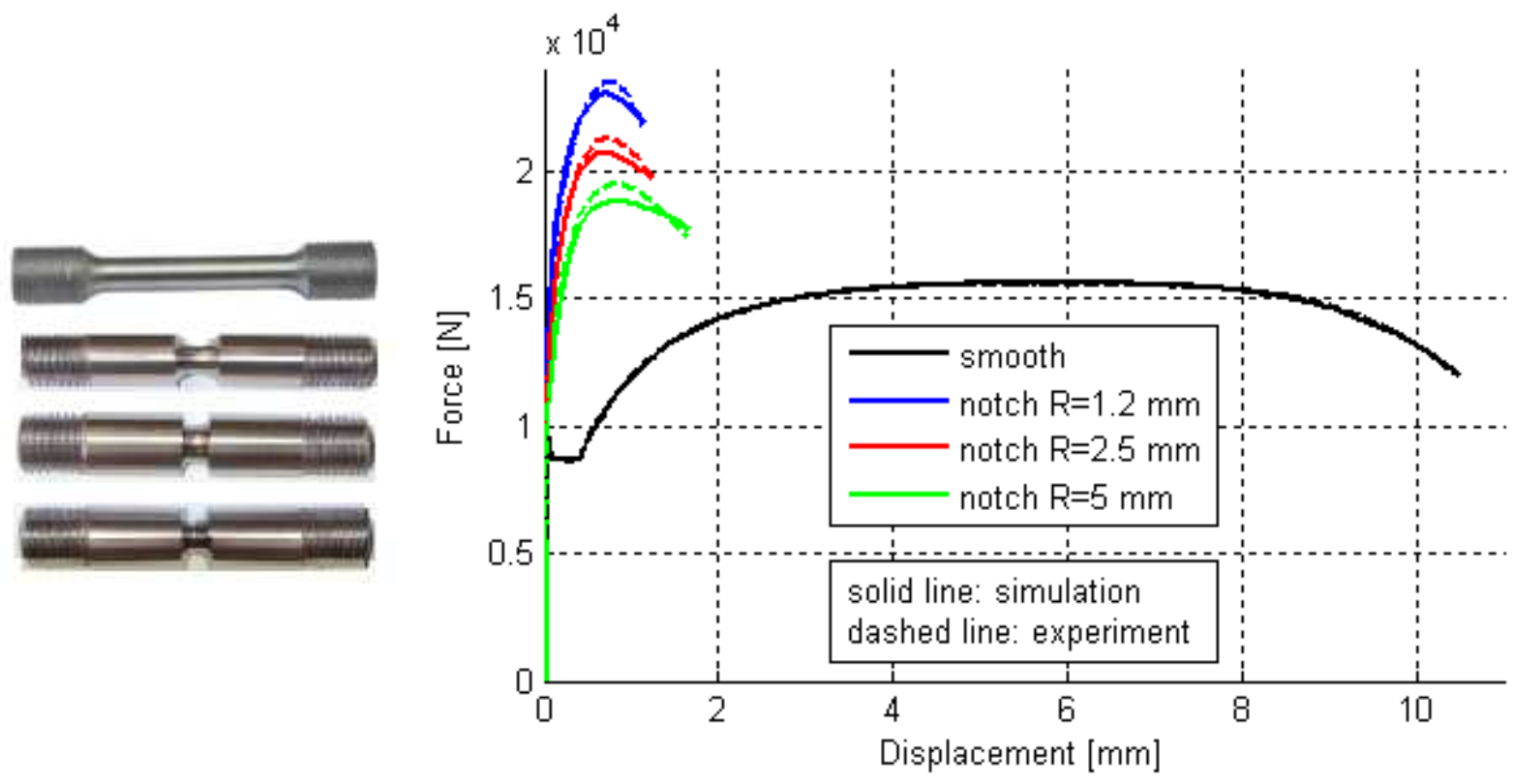

Fig.1 Tensile tests specimen and results

Calibration of the criteria parameters was realized by simulation of the tensile tests up to the fracture. From the fracture initiation point, which was located in all the cases at the symmetry axis, detailed history of stress and strain evolution can be obtained from the simulation results. This can be 
seen in Fig. 2 as blue, yellow and violet curves for different specimen notch radius. Insertion of these data into the equations defining the fracture criteria, unknown parameters of the fracture criteria can be evaluated. As there are more tests than the number of identified parameters, a least square technique is used for minimization the difference between computationally evaluated and experimentally obtained fracture point. Values of the calibrated parameters are given in Table 2 . Limit fracture strain curves for all criteria can be seen in Fig.2, too. Typical growth of fracture strain for compressive stress states with negative stress triaxiality is visible for all the criteria used here. We may also note that each stress-strain path of individual specimen has its end (fracture point) very close to the limit strain curve, especially if a mean value of triaxiality during the specimen loading is taken into the account - see the dashed lines in Fig.2.

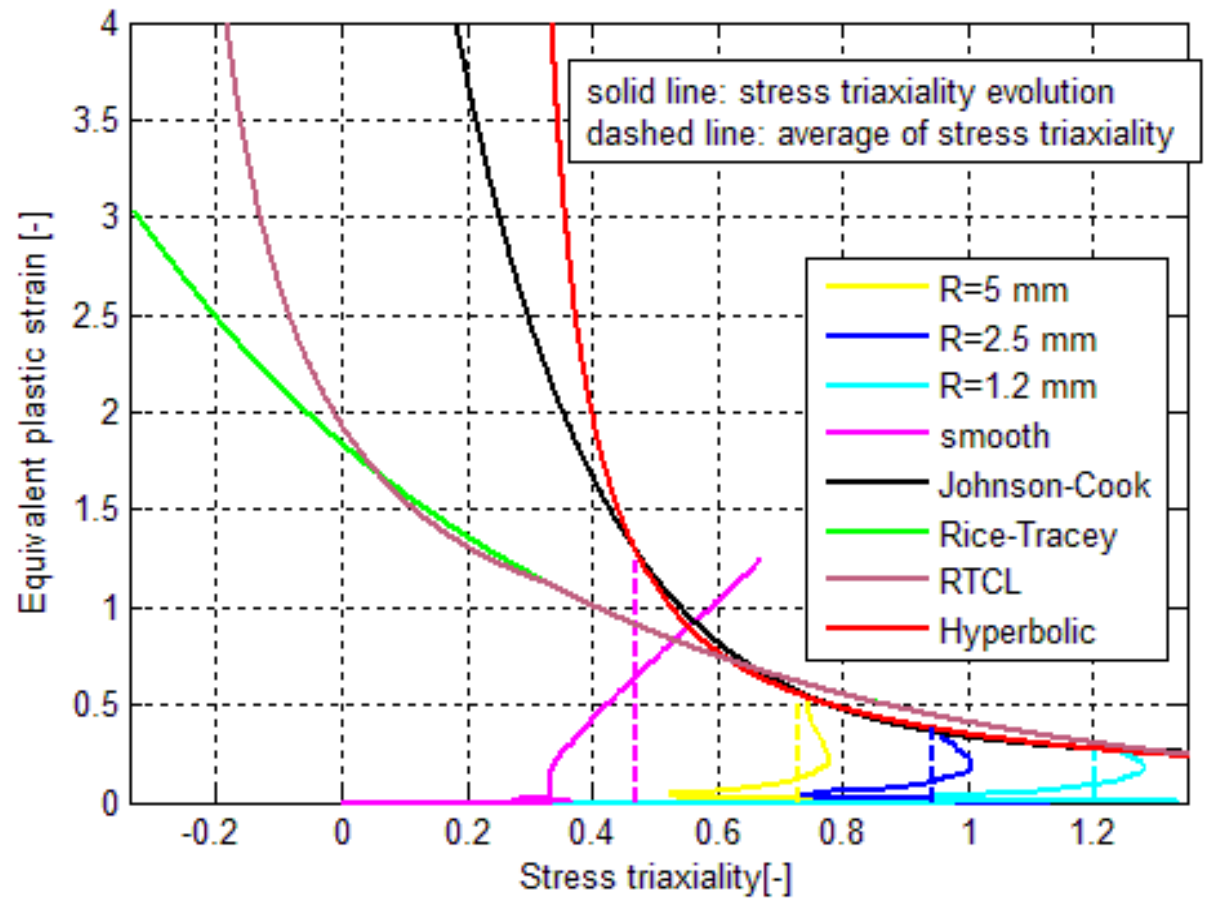

Fig.2 Stress-strain evolution in fracture locus of each specimen and fracture strain limit curves

Table 2 Calibrated parameters of the fracture criteria

\begin{tabular}{|c|c|c|c|}
\hline criterion & \multicolumn{3}{|c|}{ constants } \\
\hline Rice-Tracey & \multicolumn{3}{|c|}{$D_{R T}=1,117$} \\
\hline RTCL & \multicolumn{3}{|c|}{$D_{R T C L}=1,117$} \\
\hline Hyperbolic & $E_{1}=-0.27$ & $E_{2}=0.253$ \\
\hline Johnson-Cook & $D_{1}=0.232$ & $D_{2}=8.465$ & $D_{3}=-4.427$ \\
\hline
\end{tabular}

\section{Forward extrusion experiments and simulation}

Based on the calibrated criteria, a sequence of forward extrusion reductions of a rod with circular cross section was suggested starting from the initial diameter $27 \mathrm{~mm}$. Six subsequent reductions with final diameter $13.2 \mathrm{~mm}$ were realized, first as a computational simulation and then in reality in cooperation with our industrial partner. Correspondence of fracture initiation and growth in our computational models and experimental specimens was the aim of our investigation. To avoid the FE mesh dependence of the computational results, the mesh density was kept at the same level for extrusion as it had been during the tensile testing simulation $(0.075 \mathrm{~mm}$ element length). Totally six reductions were simulated using an axisymmetrical model of extruded material (Fig.3) with the six rigid extrusion dies in a single sequence. The material was pushed through all the dies with Coulomb friction coefficient 0.1 between the die and material prescribed at the positions of conical parts of the dies and their close vicinity (red lines showing the die surface in Fig.3). 


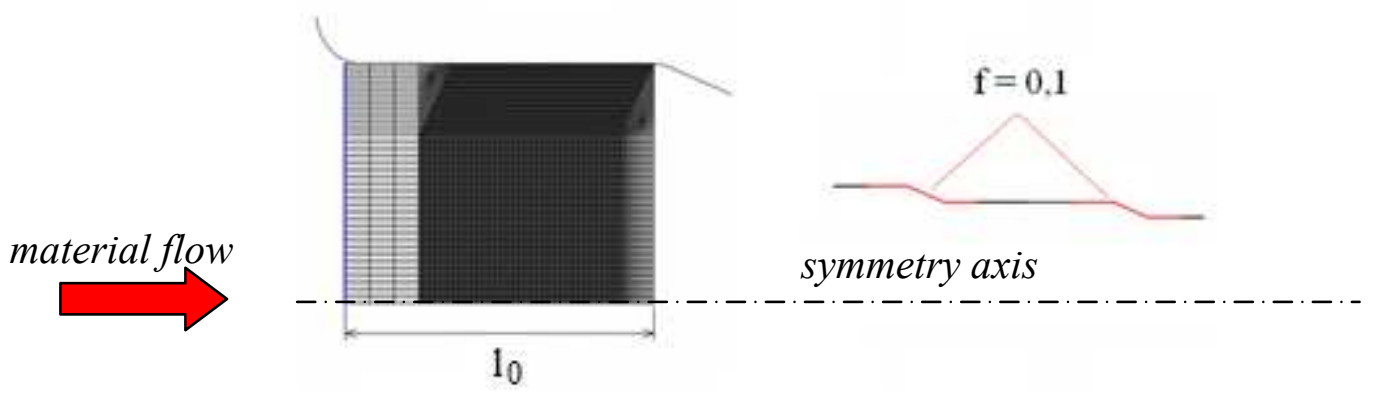

Fig.3 FE model of the extrusion

All the dies had their conical parts with the semiangle $20^{\circ}$. The simulation was realized by Abaqus explicit code with fracture process modeled by deletion of elements, where the damage cumulation reached critical level. This was realized by user subroutine VUMAT, implemented to Abaqus.

Experimental results are presented in Fig. 4 as X-ray pictures of internal damage of six specimens, extruded in the same sequence of reductions as in computations. We can see there is no fracture in the fifth reduction, but all the specimens have typical chevron cracks after the sixth reduction. Typically, 4-5 fractures can be found in the extruded end of the semiproduct.

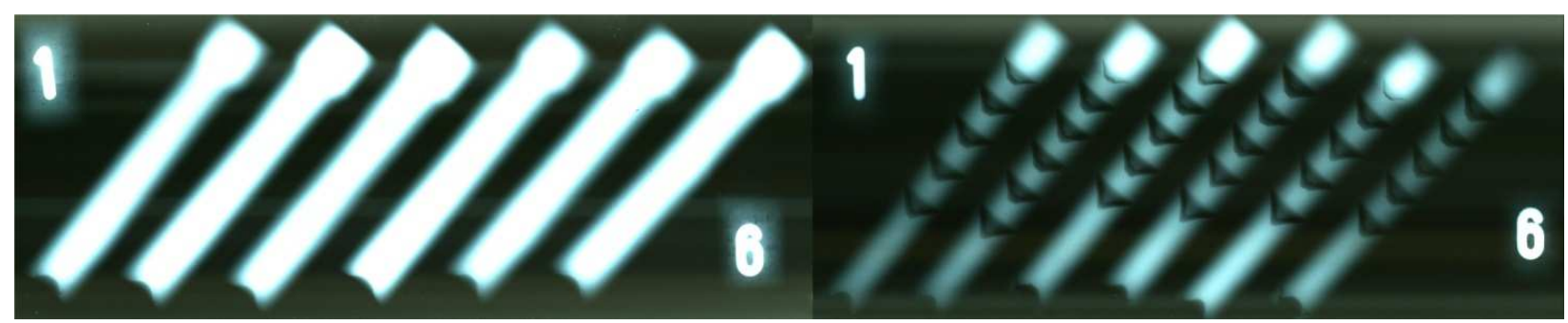

Fig. 4 X-ray of internal damage of extruded specimens after $5^{\text {th }}$ (left) and $6^{\text {th }}$ (right) reduction

Computational results in Fig.5a-d show the prediction of chevron cracks by ductile fracture criteria described in the previous part of this paper. We can identify clear distinctions between the experimental and computational results. Hyperbolic and Johnson-Cook criteria predict a fine sequence of relatively small cracks in the central zone of the extruded rod. They do not grow in a typical arrow-like shape close to external surface of the extruded rod as can be seen in Fig.4. For Rice-Tracey and RTCL criterion, the arow-like shape is manifested much better, but still the cone angle of the fracture is lower than real shape in Fig.4 and the damaged zone is again limited to close vicinity of the symmetry axis.

Discussion. The results show that the applied criteria are able to predict initiation of chevron cracks during forward extrusion, but are not successful in correct prediction of further propagation and final shape of the cracks. The reason is that the fracture is initiated at symmetry axis, where a close similarity exists between actual stress state and the stress state of tensile tests used for calibration. During propagation, the fracture moves to area with dominant shear stress, which is not correctly described by the applied fracture criteria. The difference can be quantified by the Lode parameter, or normalized third invariant $\xi$ of stress deviator $\mathrm{J}_{3}$

$$
\xi=\frac{27}{2} \frac{J_{3}}{\sigma_{e q}{ }^{3}}
$$

The value of $\xi$ is continuously changed from 1 at the symmetry axis to 0 in the area with dominant shear. Using generalized fracture criterion according to [7], simple hyperbolic criterion described by Eq.6 (green surface in Fig.6) was extended to incorporate $\xi$ as an independent variable (pink surface in Fig.6). Results of computational simulation of extrusion with the generalized hyperbolic criterion show, that now the criterion is able to predict correctly both the initiation and growth of chevron cracks (Fig.7). To calibrate the generalized criterion correctly, more specimens should be now tested to cover set of stress states with various levels of $\xi$. This problem can be solved using notched tube specimens loaded by combination of tension and torsion as suggested in [8]. 


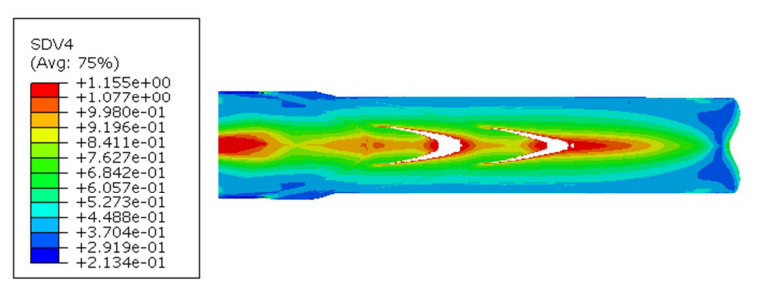

Fig.5a Fracture by Rice-Tracey criterion
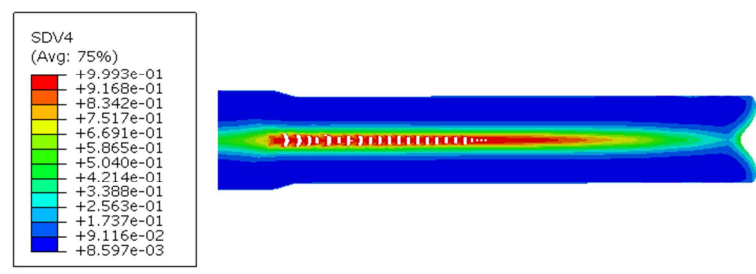

Fig.5c Fracture by Johnson-Cook criterion

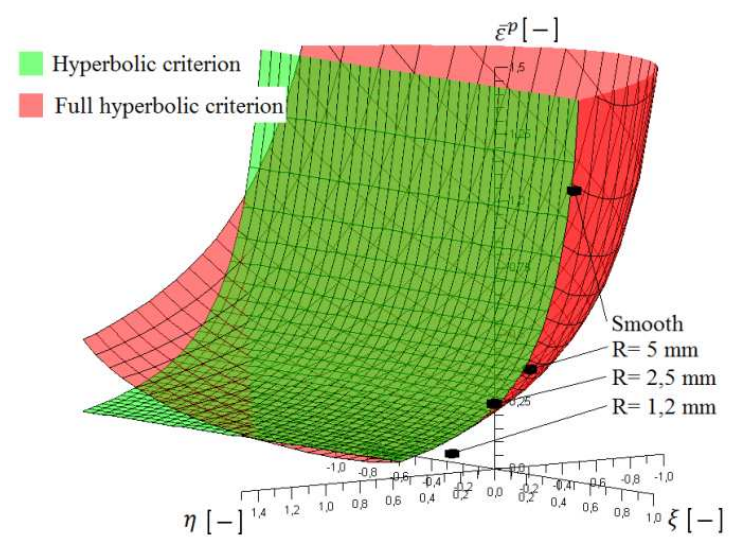

Fig. 6 Generalized (full) hyperbolic criterion
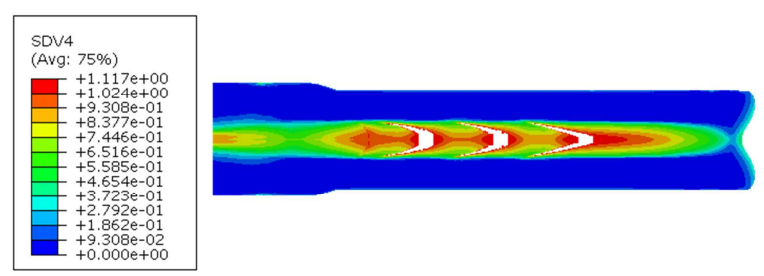

Fig.5b Fracture predicted by RTCL criterion
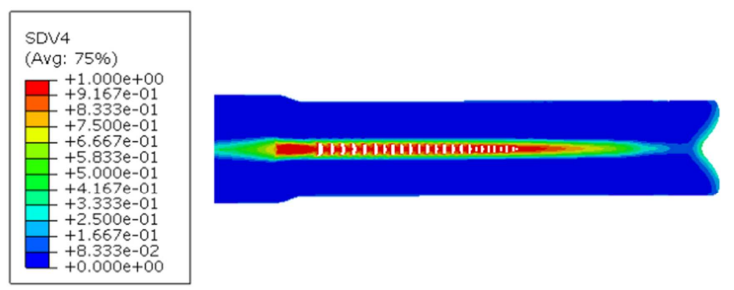

Fig.5d Fracture predicted by hyperbolic criterion
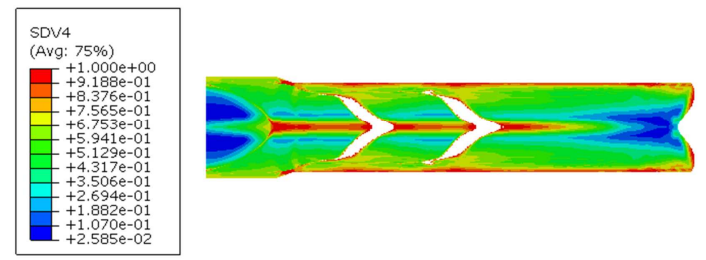

Fig.7 Fracture predicted by generalized hyperbolic criterion

\section{Summary}

Four ductile fracture criteria based on stress triaxiality were calibrated and applied to prediction of chevron crack during forward extrusion. The results show good predictive capacity of the criteria with respect to fracture initiation. Nevertheless, to predict the fracture growth correctly, general criteria should be applied with triaxiality and Lode parameter as independent variables. One example of such criterion was successfully applied to complex simulation of sequential extrusion of rods with circular cross section. Further work is necessary to develop efficient methods to calibrate such general fracture criteria to make them applicable for industrial practice.

\section{Acknowledgement}

Financial help of the grant projects GA101/09/1630, FSI-S-11-11/1190 and European Regional Development Fund project CZ.1.05/2.1.00/01.0002 is gratefully acknowledged 


\section{References}

[1] K. Saanouni, J.F. Mariage, A. Cherouat, P. Lestriez: Computers and Structures, Vol. 82 (2004), pp. 2309-2332

[2] C. McVeigh, W.K. Liu: Int. J. Solids and Structures, Vol. 43 (2006), pp. 3087-3105

[3] J.R. Rice, D.M. Tracey: J. Mech. and Physics of Solids, Vol. 17 (1969), 201-217

[4] R. Törnqvist: Design of Crashworthy Ship Structures, (PhD Thesis, Technical University of Denmark, 2003)

[5] R. Johnson and W.H. Cook: Eng. Fract. Mech., Vol. 21 (1985), 31-48

[6] G. Mirone: Engineering Fracture Mechanics, Vol. 74 (2006), pp. 1203-1221

[7] Bai, Y., Wierzbicki, T.: Int. J. of Plasticity, Vol. 24 (2008), pp. 1071-1096

[8] J. Hulka, P. Kubik, J. Petruska: Adv. Mat. Research, Vols. 468-471 (2012), pp. 1049-1052 